01

\title{
Высокочастотный магнетотранспорт в тонком металлическом слое С варьируемыми коэффициентами зеркальности границ
}

\author{
(C) П.А. Кузнецов, О.В. Савенко, А.А. Юшканов \\ Ярославский государственный университет им. П.Г. Демидова, \\ 150003 Ярославль, Россия \\ e-mail: o.savenko@uniyar.ac.ru
}

Поступило в Редакцию 28 января 2020 г.

В окончательной редакции 3 апреля 2020 г.

Принято к публикации 7 апреля 2020 г.

Построена теоретическая модель электропроводности тонкого металлического слоя в продольном постоянном магнитном и переменном электрическом полях с учетом диффузно-зеркальных граничных условий. Получено аналитическое выражение для интегральной проводимости, как функции безразмерных параметров: толщины слоя, частоты электрического поля, индукции магнитного поля и коэффициентов зеркальности поверхностей. Проанализированы зависимости проводимости слоя от вышеуказанных параметров. Проведено сравнение полученных результатов с известными экспериментальными данными.

Ключевые слова: уравнение Больцмана, функция распределения, тонкий слой, электропроводность, коэффициент зеркальности.

DOI: $10.21883 /$ JTF.2020.12.50114.36-20

\section{Введение}

Исследования электрических, оптических и гальваномагнитных свойств тонких проводящих слоев связаны прежде всего с бурным развитием микро-, нано- и оптоэлектроники в последние десятилетия. Тонкие слои используются как основа всех полупроводниковых приборов и интегральных схем, применяются для создания многослойных солнечных элементов, в настоящее время ведутся активные разработки и исследования по увеличению их КПД и энергоэффективности [1-5]. Имеется немало работ, посвященных применению тонких слоев в фотонике и нанофотонике [6,7], СВЧ-электронике $[8,9]$ и т.д.

Известно, что электропроводность тонкого слоя меньше электропроводности макроскопического образца. Среди причин, объясняющих это различие, помимо квантовых размерных эффектов, могут быть явления, имеющие классическое объяснение. При комнатной температуре во многих типичных металлах средняя длина свободного пробега носителей заряда лежит в пределах 10-100 nm, а в типичных полупроводниках $10-1000 \mathrm{~nm}$ [10,11]. В случае, когда толщина слоя много больше длины волны де Бройля носителя заряда, принимающей значение порядка межатомного расстояния $(\sim 0.3 \mathrm{~nm})$ для металлов и порядка $10 \mathrm{~nm}$ для полупроводников [10,11], справедливо классическое кинетическое описание размерных эффектов. Современные технологии позволяют создавать элементы интегральных схем с характерным размером порядка нанометров. Поэтому ситуация, когда для решения задач необходимо учитывать поверхностное рассеяние носителей заряда, но можно пренебречь квантовыми эффектами, реализуется на практике.
Теоретические исследования электрических свойств тонких проводящих пленок проводятся с первой половины XXв. В работе [12] для решения задачи о статической проводимости тонкой металлической пленки впервые использована модель диффузно-зеркальных граничных условий. В рамках данной модели было введено понятие коэффициента зеркальности поверхности, характеризующего относительное число электронов, отражающихся от поверхности зеркально. В работах $[13,14]$ решены задачи о статической проводимости тонкой металлической пленки в продольном и перпендикулярном магнитном поле. Имеются публикации, посвященные изучению процессов переноса, происходящих в тонких проволоках. Так, в работе [15] построена теоретическая модель электропроводности тонкой проволоки в продольном магнитном поле с учетом диффузных граничных условий. Эта теоретическая модель была использована в работах $[16,17]$ для сравнения с экспериментальными зависимостями сопротивления тонких проволок меди от индукции магнитного поля температуре $4.2 \mathrm{~K}$. Согласие теории с экспериментом наблюдалось только в области слабых магнитных полей $(B<0.2 \mathrm{~T})$. Авторы работы [17] связывали расхождение экспериментальных и теоретических данных с несферичностью поверхности Ферми меди, поскольку в работе [15] анизотропия изоэнергетической поверхности не учитывалась. Кроме того, к расхождению результатов могло приводить ограничение модели поверхностного рассеяния рассмотренным в работе [15] частным случаем диффузного рассеяния носителей заряда [15]. Кинетический расчет электропроводности тонкой проволоки в продольном магнитном поле с учетом диффузно-зеркальных граничных условий представлен в работе [18]. Сравнение теоретических расчетов [18] с экспериментальными данными [17] по- 
казало, что хорошего согласия (отличие меньше 6\%) можно добиться при одинаковом для всех проволок коэффициенте зеркальности, равным 0.12, и различных значениях длин свободного пробега носителей заряда.

Теоретические исследования электрических свойств тонких проводящих пленок и проволок продолжаются в настоящее время. В работе [19] решена задача о статической проводимости тонкой металлической поликристаллической пленки. Работы [20-22] посвящены изучению влияния магнитного поля различной ориентации по отношению к плоскости пленки на удельное сопротивление тонких пленок золота зернистой структуры при температурах 4-50 К. Случай переменного электрического поля рассматривался в работах [23-29]. В работах [23-26] решены задачи об электропроводности тонкой металлической пленки в отсутствие и при наличии перпендикулярного магнитного поля. В работе [27] построено теоретическое решение задачи об электропроводности тонкой металлической пленки с учетом эллипсоидальной формы поверхности Ферми. В работах $[28,29]$ построены теоретические модели взаимодействия электромагнитной $E$ - и $H$-волны с тонкой металлической пленкой. В работах [30,31] решены задачи об электропроводности и самоиндукции тонкой металлической проволоки с учетом сферическисимметричной [30] и эллипсоидальной [31] формы изоэнергетической поверхности.

В настоящей работе решена кинетическая задача об электропроводности тонкого металлического слоя в продольном постоянном магнитном и переменном электрическом поле с учетом модели Фукса и различных коэффициентов зеркальности поверхностей.

\section{1. Постановка задачи}

Рассмотрим тонкий слой толщины $a$, материалом которого является металл или сильно легированный полупроводник $n$ - или $p$-типа проводимости (рис. 1). Слой расположен в продольном постоянном магнитном поле индукции В и переменном электрическом поле напряженности Е. Предполагается, что толщина слоя

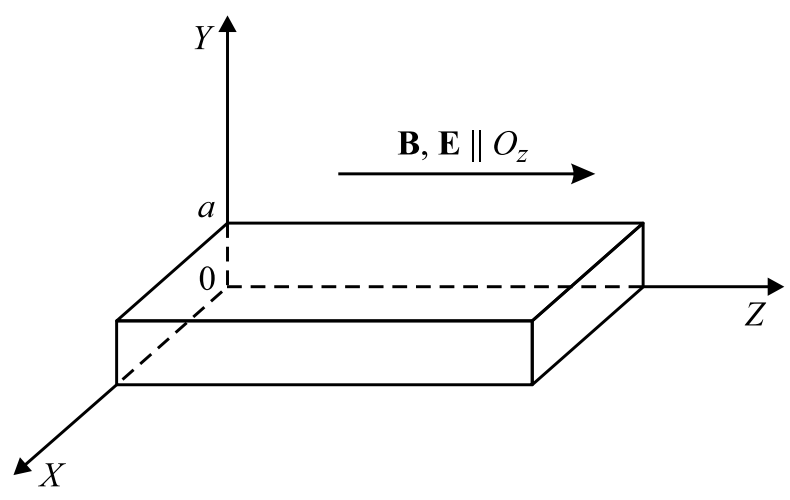

Рис. 1. Тонкий металлический слой в продольном магнитном и электрическом полях. много меньше глубины скин-слоя, что позволяет пренебречь скин-эффектом.

Однородное периодическое по времени электрическое поле изменяется по закону (1):

$$
\mathbf{E}=\mathbf{E}_{0} \exp (-i \omega t)
$$

где $\omega$ - частота электрического поля.

Электрическое поле действует на электроны проводимости, вызывая отклонение $f_{1}$ их функции распределения $f$ от равновесной функции Ферми-Дирака $f_{0}$ :

$$
\begin{aligned}
f(\mathbf{v}, y, t)= & f_{0}(\varepsilon)+f_{1}(\mathbf{v}, y, t)=f_{0}(\varepsilon)+f_{1}(\mathbf{v}, y) \\
& \times \exp (-i \omega t) .
\end{aligned}
$$

Равновесная функция распределения имеет вид ступенчатой аппроксимации:

$$
f_{0}(\varepsilon)= \begin{cases}1 & 0<\varepsilon<\varepsilon_{F}, \\ 0, & \varepsilon>\varepsilon_{\mathrm{F}},\end{cases}
$$

где $\varepsilon=\frac{m v^{2}}{2}-$ кинетическая энергия электрона в случае сферически-симметричной энергетической зоны, $m$ и $v$ - соответственно масса и скорость электрона, $\varepsilon_{\mathrm{F}}=m v_{\mathrm{F}}^{2} / 2-$ энергия Ферми, $v_{\mathrm{F}}-$ скорость Ферми, $y$ - координата по оси $Y$.

Функция $f_{1}$ подчиняется кинетическому уравнению Больцмана в приближении времени релаксации $\tau$ и в линейном по внешнему полю приближении [13]:

$$
-i \omega f_{1}+v_{y} \frac{\partial f_{1}}{\partial y}-e(\mathbf{v E}) \frac{\partial f_{0}}{\partial \varepsilon}-\frac{e}{m}[\mathbf{v}, \mathbf{B}] \frac{\partial f_{1}}{\partial \mathbf{v}}=-\frac{f_{1}}{\tau} .
$$

Здесь $e-$ элементарный заряд.

Поскольку вектор индукции магнитного поля направлен параллельно напряженности электрического поля, четвертое слагаемое в уравнении (4) равно нулю. Из выражения (3) следует, что

$$
\frac{\partial f_{0}}{\partial \varepsilon}=-\delta\left(\varepsilon-\varepsilon_{\mathbf{F}}\right)=-\frac{\delta\left(v-v_{\mathrm{F}}\right)}{m v_{\mathrm{F}}} .
$$

Кинетическое уравнение (4) с учетом вышесказанного будет иметь вид

$$
v f_{1}+v_{y} \frac{\partial f_{1}}{\partial y}+\frac{e v_{z} E}{m v_{\mathrm{F}}} \delta\left(v-v_{\mathrm{F}}\right)=0 .
$$

Здесь $v=\tau^{-1}-i \omega-$ комплексная частота рассеяния.

В качестве граничных условий к уравнению (6) используется модель Фукса. Коэффициенты зеркальности верхней и нижней поверхности слоя предполагаются разными. Отметим, что проекция траектории электрона на плоскость, перпендикулярную направлению индукции магнитного поля, т. е. на поперечное сечение слоя, будет иметь вид окружности. Поэтому реализуется ситуация, когда траектория носителя заряда может пересекать границу слоя, а может и не пересекать. В этом случае 

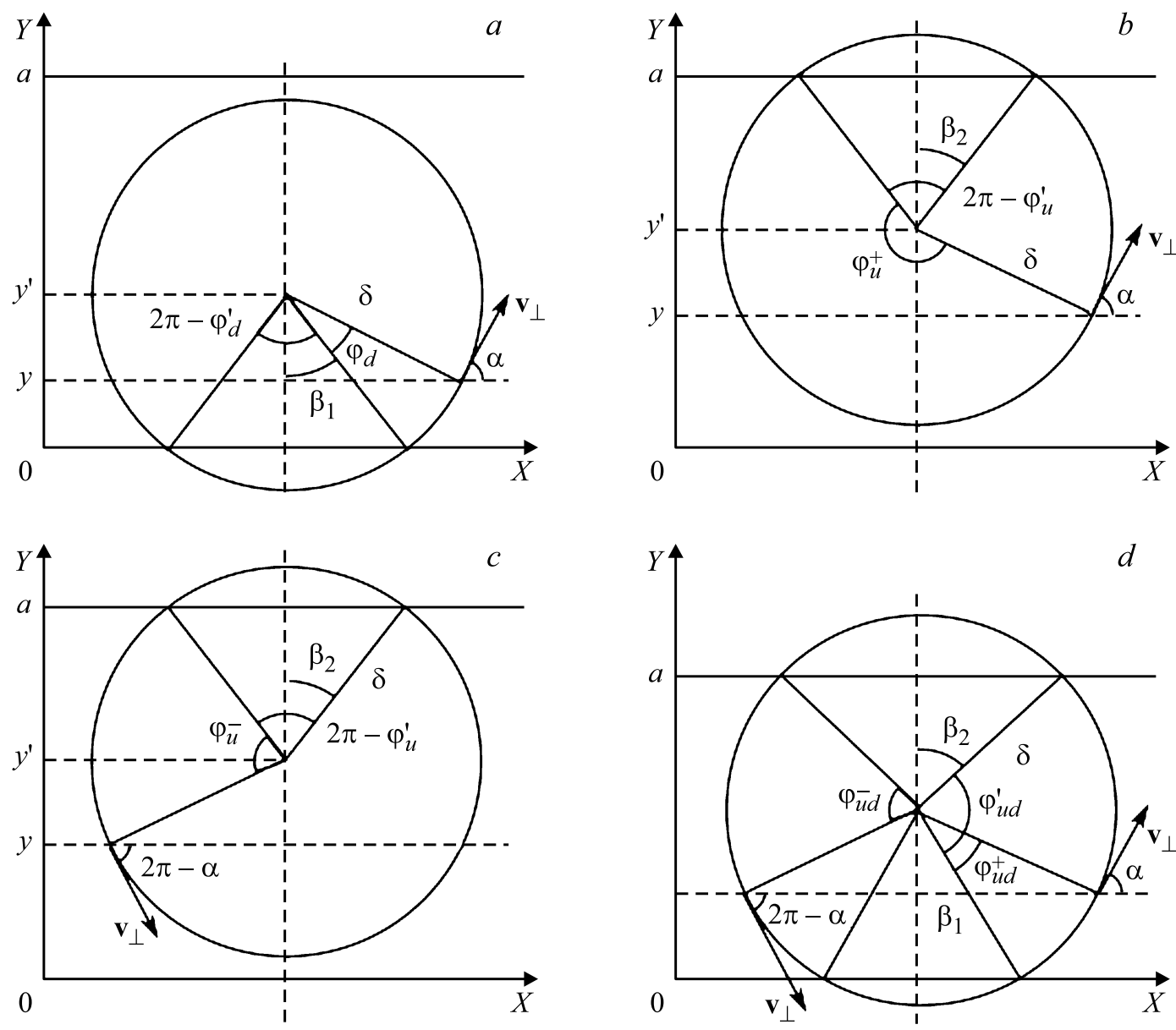

Рис. 2. Поперечное сечение слоя и траектория носителя заряда, рассеивающегося только на нижней поверхности $(a)$; только на верхней поверхности при $\alpha \in(0 ; \pi)(b)$, при $\alpha \in(\pi ; 2 \pi)(c)$ и на обоих поверхностях слоя $(d)$.

носители заряда в слое можно разделить на четыре типа: носители, рассеивающиеся только на нижней поверхности, только на верхней поверхности, сразу на обеих поверхностях слоя и вообще не испытывающие рассеяние.

Пусть $f_{1}^{d}, f_{1}^{u}, f_{1}^{u d}$ и $f_{1}^{v}$ - соответственно неравновесные поправки к функции распределения носителей заряда первого, второго, третьего и четвертого типов. Тогда граничные условия для этих функций (рис. 2) будут иметь следующий вид:

$$
\begin{gathered}
f_{1}^{d}\left(v_{y}, 0\right)=q_{1} f_{1}^{d}\left(-v_{y}, 0\right), \\
f_{1}^{u}\left(-v_{y}, a\right)=q_{2} f_{1}^{u}\left(v_{y}, a\right), \\
\left\{\begin{array}{l}
f_{1}^{u d}\left(v_{y}, 0\right)=q_{1} f_{1}^{u d}\left(-v_{y}, 0\right), \\
f_{1}^{u d}\left(-v_{y}, a\right)=q_{2} f_{1}^{u d}\left(v_{y}, a\right) .
\end{array}\right.
\end{gathered}
$$

Пусть $y^{\prime}$ - координата центра окружности, представляющей собой проекцию траектории носителя заряда на поперечное сечение слоя, $\delta=m v_{\perp} / e B-$ радиус окружности. Из рис. 2 следует, что траектория носителя заряда пересекает нижнюю и верхнюю поверхности слоя при соответствующих условиях

$$
y^{\prime}<\delta, \quad a-y^{\prime}<\delta .
$$

Искомую функцию можно записать в виде

$$
\begin{aligned}
f_{1}= & \Theta\left(A_{1}\right) \Theta\left(-A_{2}\right) f_{1}^{d}+\Theta\left(-A_{1}\right) \Theta\left(A_{2}\right) f_{1}^{u} \\
& +\Theta\left(A_{1}\right) \Theta\left(A_{2}\right) f_{1}^{u d}+\Theta\left(-A_{1}\right) \Theta\left(-A_{2}\right) f_{1}^{v}
\end{aligned}
$$

где введены следующие обозначения:

$$
A_{1}=1-\frac{y^{\prime}}{\delta}, \quad A_{2}=1-\frac{a-y^{\prime}}{\delta} .
$$

Неравновесная функция распределения позволяет рассчитать плотность тока в слое, которая определяется стандартным выражением

$$
\mathbf{j}=-2 e\left(\frac{m}{h}\right)^{2} \int \mathbf{v} f_{1} d^{3} v
$$

Здесь $h-$ постоянная Планка.

Таким образом, решая кинетическое уравнение (6) с учетом граничных условий $(7)-(9)$, можно получить выражения для неравновесных функций распределения носителей заряда, которые позволят рассчитать плотность тока и интегральную проводимость. 


\section{2. Метод решения и математические расчеты}

Заметим, что плотность тока можно записать как сумму составляющих плотности тока, учитывающих рассеяние носителей заряда только на нижней поверхности, на верхней поверхности, на обеих поверхностях и объемное рассеяние носителей заряда:

$$
\mathbf{j}=\mathbf{j}^{d}+\mathbf{j}^{u}+\mathbf{j}^{u d}+\mathbf{j}^{v}
$$

где каждая составляющая тока определяется выражением (13), а вместо функции $f_{1}$ фигурирует соответствующее слагаемое, стоящее в выражении (11).

Найдем составляющую плотности тока $\mathrm{j}^{d}$. Кинетическое уравнение (6) с учетом граничного условия (7) решается методом, аналогичным работе [32]. Неравновесная функция распределения носителей заряда, рассеивающихся на нижней поверхности слоя, будет иметь вид

$$
f_{1}^{d}\left(t^{\prime}\right)=-\frac{e v_{z} E}{v m v_{\mathrm{F}}} \delta\left(v-v_{\mathrm{F}}\right)\left[1-\frac{\left(1-q_{1}\right) \exp \left(-v t^{\prime}\right)}{1-q_{1} \exp \left(-v T^{\prime}\right)}\right] .
$$

Здесь $t^{\prime}$ - время движения электрона вдоль траектории от границы, на которой происходит отражение, до текущей точки, $T^{\prime}$ - время движения электрона между двумя последовательными столкновениями с границей слоя.

В случае, когда траектория носителя заряда пересекает поверхность слоя, параметры $t^{\prime}$ и $T^{\prime}$ выражаются следующим образом:

$$
t^{\prime}=\varphi \delta / v_{\perp}, \quad T^{\prime}=\varphi^{\prime} \delta / v_{\perp}
$$

где $\varphi$ и $\varphi^{\prime}-$ соответственно центральные углы дуг, соответствующих траектории движения носителя заряда от точки соударения с поверхностью до текущей точки и между двумя последовательными соударениями с поверхностью слоя.

Для расчета плотности тока удобно воспользоваться цилиндрической системой координат, главная ось которой сонаправлена с направлением вектора индукции магнитного поля $\left(\mathbf{v}=\left(\mathbf{v}_{1}, \alpha, \mathbf{v}_{z}\right), \mathbf{v}_{z}-\right.$ полярная ось):

$j_{z}^{d}=-2 e\left(\frac{m}{h}\right)^{3} \int_{-\infty}^{\infty} \int_{0}^{\infty} \int_{0}^{2 \pi} v_{z} \Theta\left(A_{1}\right) \Theta\left(-A_{2}\right) f_{1}^{d} v_{\perp} d v_{\perp} d \alpha d v_{z}$

Отметим, что в силу симметрии задачи интегрирование по $v_{z}$ заменяется интегрированием по положительному диапазону, и результат удваивается. Подставляя выражение для неравновесной функции распределения (15) в (17) и учитывая вышесказанное, получим следующее выражение:

$$
\begin{aligned}
j_{z}^{d} & =\frac{4 e^{2} E}{v m}\left(\frac{m}{h}\right)^{3} \int_{0}^{\infty} \int_{0}^{\infty} \int_{0}^{2 \pi} \frac{v_{\perp} v_{z}^{2}}{\sqrt{v_{\mathrm{F}}^{2}-v_{\perp}^{2}}} \delta\left(v_{z}-\sqrt{v_{\mathrm{F}}^{2}-v_{\perp}^{2}}\right) \\
& \times \Theta\left(A_{1}\right) \Theta\left(-A_{2}\right)\left[1-\frac{\left(1-q_{1}\right) \exp \left(-v t^{\prime}\right)}{1-q_{1} \exp \left(-v T^{\prime}\right)}\right] d v_{\perp} d \alpha d v_{z} .
\end{aligned}
$$

Локальную проводимость слоя можно найти, используя локальный закон Ома:

$$
\sigma_{d}=j_{z}^{d} / E
$$

Проинтегрировав выражение (19) по координате $y$, получим полную интегральную проводимость, учитывающую рассеяние электронов на нижней поверхности слоя:

$$
\begin{aligned}
& \sigma_{a d}=\int_{0}^{a} \sigma_{d} d y=\frac{3}{2 \pi} \frac{a \sigma_{0} x_{0}}{z_{0}} \int_{0}^{1} \int_{0}^{1} \int_{0}^{2 \pi} \rho \sqrt{1-\rho^{2}} \\
& \times \Theta\left(A_{1}\right) \Theta\left(-A_{2}\right)\left[1-\frac{\left(1-q_{1}\right) \exp \left(-z_{0} \varphi_{d} / \beta_{0}\right)}{1-q_{1} \exp \left(-z_{0} \varphi_{d}^{\prime} / \beta_{0}\right)}\right] d \rho d \alpha d \xi .
\end{aligned}
$$

Введены безразмерные параметры:

$$
\begin{gathered}
z_{0}=\frac{a v}{v_{\mathrm{F}}}=\frac{a}{v_{\mathrm{F}} \tau}-i \frac{a \omega}{v_{\mathrm{F}}}=x_{0}-i y_{0}, \\
\beta_{0}=\frac{e B a}{m v_{\mathrm{F}}}, \quad \xi=\frac{y}{a}, \quad \rho=\frac{v_{\perp}}{v_{\mathrm{F}}} .
\end{gathered}
$$

Здесь $\sigma_{0}=\frac{n e^{2} \tau}{m}-$ статическая проводимость, $\varphi_{d}$ и $\varphi_{d}^{\prime}-$ соответственно центральные углы дуг, соответствующих траектории движения электрона от точки соударения с нижней поверхностью слоя до текущей точки и между двумя последовательными соударениями с нижней поверхностью.

Аналогично решается задача о нахождении составляющей проводимости слоя, учитывающей столкновения носителей заряда с верхней поверхностью слоя. Решение уравнения Больцмана (6) с учетом условия (8) имеет следующий вид:

$$
f_{1}^{u}\left(t^{\prime}\right)=-\frac{e v_{z} E}{v m v_{\mathrm{F}}} \delta\left(v-v_{\mathrm{F}}\right)\left[1-\frac{\left(1-q_{2}\right) \exp \left(-v t^{\prime}\right)}{1-q_{2} \exp \left(-v T^{\prime}\right)}\right] .
$$

Подставляя выражение (22) в (13) и проведя аналогичные предыдущему случаю вычисления, получим выражение

$$
\begin{aligned}
& \sigma_{a u}=\frac{3}{2 \pi} \frac{a \sigma_{0} x_{0}}{z_{0}} \int_{0}^{1} \int_{0}^{1} \int_{0}^{2 \pi} \rho \sqrt{1-\rho^{2}} \\
& \times \Theta\left(-A_{1}\right) \Theta\left(A_{2}\right)\left[1-\frac{\left(1-q_{2}\right) \exp \left(-z_{0} \varphi_{u} / \beta_{0}\right)}{1-q_{2} \exp \left(-z_{0} \varphi_{u}^{\prime} / \beta_{0}\right)}\right] d \rho d \alpha d \xi .
\end{aligned}
$$


Здесь $\varphi_{u}$ и $\varphi_{u}^{\prime}-$ соответственно центральные углы дуг, соответствующих траектории движения носителя заряда от точки соударения с верхней поверхностью слоя до текущей точки и между двумя последовательными соударениями с верхней поверхностью.

Найдем составляющую проводимости слоя, учитывающую столкновения носителей заряда с обоими поверхностями слоя. Решая уравнение Больцмана (6) с учетом граничных условий (9), получим выражения для функции распределения $f_{1}^{u d}$ :

$$
\begin{aligned}
& f_{1}^{u d}\left(t^{\prime}\right)= \\
& \left\{\begin{array}{r}
-\frac{e v_{z} E}{v m v_{\mathrm{F}}} \delta\left(v-v_{\mathrm{F}}\right)\left[1-\frac{1-q_{1}+q_{1}\left(1-q_{2}\right) \exp \left(-v T^{\prime}\right)}{1-q_{1} q_{2} \exp \left(-2 v T^{\prime}\right)}\right. \\
\left.\quad \times \exp \left(-v t^{\prime}\right)\right]\left(v_{y}>0\right), \\
-\frac{e v_{z} E}{v m v_{\mathrm{F}}} \delta\left(v-v_{\mathrm{F}}\right)\left[1-\frac{1-q_{2}+q_{2}\left(1-q_{1}\right) \exp \left(-v T^{\prime}\right)}{1-q_{1} q_{2} \exp \left(-2 v T^{\prime}\right)}\right. \\
\left.\times \exp \left(-v t^{\prime}\right)\right]\left(v_{y}<0\right),
\end{array}\right.
\end{aligned}
$$

Проведя аналогичные вычисления, получим следующее выражение для интегральной проводимости:

$$
\begin{gathered}
\sigma_{a u d}=\frac{3}{2 \pi} \frac{a \sigma_{0} x_{0}}{z_{0}} \int_{0}^{1} \int_{0}^{1} \rho \sqrt{1-\rho^{2}} \Theta\left(A_{1}\right) \Theta\left(A_{2}\right) \\
\times\left[2 \pi-\int_{0}^{\pi} \psi_{1}\left(\varphi_{u d}^{+}, \varphi_{u d}^{\prime+}\right) d \alpha-\int_{\pi}^{2 \pi} \psi_{2}\left(\varphi_{u d}^{-}, \varphi_{u d}^{\prime-}\right) d \alpha\right] d \rho r \xi \\
\psi_{1}\left(\varphi, \varphi^{\prime}\right)= \\
\quad \times \frac{\exp \left(-z_{0} \varphi / \beta_{0}\right),}{1-q_{1} q_{2} \exp \left(-2 z_{0} \varphi^{\prime} / \beta_{0}\right)} \\
\psi_{2}\left(\varphi, \varphi^{\prime}\right)=\frac{1-q_{2}+q_{2}\left(1-q_{1}\right) \exp \left(-z_{0} \varphi^{\prime} / \beta_{0}\right)}{1-q_{1} q_{2} \exp \left(-2 z_{0} \varphi^{\prime} / \beta_{0}\right)} \\
\times \exp \left(-z_{0} \varphi / \beta_{0}\right) .
\end{gathered}
$$

Здесь $\varphi_{u d}^{ \pm}$и $\varphi_{u d}^{\prime \pm}-$ соответственно центральные углы дуг, соответствующих траектории движения носителя заряда от точки соударения с нижней $\left(\varphi_{u d}^{+}\right)$или верхней $\left(\varphi_{u d}^{-}\right)$поверхностью слоя до текущей точки и между двумя последовательными соударениями с верхней и нижней поверхностью слоя. Знак „+““ относится к носителям заряда, у которых $v_{y}>0$, а знак „-““ к носителям заряда, у которых $v_{y}<0$.

Теперь рассчитаем выражение для составляющей проводимости слоя, учитывающей только объемное рассеяние носителей заряда. Найдем функцию распределения $f_{1}^{v}$ не рассеивающихся на поверхностях слоя носителей.
Ее можно получить из функций $f_{1}^{u}, f_{1}^{d}$ и $f_{1}^{u d}$, если положить $t^{\prime}, T^{\prime} \rightarrow \infty$ :

$$
f_{1}^{v}=\frac{e v_{z} E}{v}\left(\frac{\partial f_{0}}{\partial \varepsilon}\right)
$$

Проводимость с учетом объемного рассеяния носителей заряда принимает вид

$$
\sigma_{a v}=\frac{3}{2 \pi} \frac{a \sigma_{0} x_{0}}{z_{0}} \int_{0}^{1} \int_{0}^{1} \int_{0}^{2 \pi} \Theta\left(-A_{1}\right) \Theta\left(-A_{2}\right) \rho \sqrt{1-\rho^{2}} d \rho d \alpha d \xi .
$$

Для численного счета интегралов (20), (23), (25) и (29) необходимо найти связь между углами $\varphi_{d}, \varphi_{u}, \varphi_{u d}^{ \pm}$, $\varphi_{d}^{\prime}, \varphi_{u}^{\prime}$ и $\varphi_{u d}^{\prime \pm}$ с переменными интегрирования. Рассмотрим поперечное сечение слоя и проекцию траектории электрона, отвечающую трем случаям рассеяния на поверхностях слоя: рассеяние только на верхней границе слоя (рис. 2, $a$ ), только на нижней границе (рис. 2, $b, c)$ и сразу на обеих границах (рис. $2, d)$.

Из геометрических построений (рис. 2) видно, что

$$
\begin{gathered}
\cos \beta_{1}=y^{\prime} / \delta=\xi \beta_{0} / \rho+\cos \alpha, \\
\cos \beta_{2}=\left(a-y^{\prime}\right) / \delta=(1-\xi) \beta_{0} / \rho-\cos \alpha, \\
\varphi_{u d}^{+}=\varphi_{d}=\alpha-\beta_{1}, \varphi_{d}^{\prime}=2 \pi-2 \beta_{1}, \varphi_{u}^{\prime}=2 \pi-2 \beta_{2}, \\
\varphi_{u}^{ \pm}=\alpha \pm \pi-\beta_{2}, \varphi_{u d}^{-}=\varphi_{u}^{-}, \varphi_{u d}^{\prime \pm}=\varphi_{u d}^{\prime}=\pi-\beta_{1}-\beta_{2} .
\end{gathered}
$$

Полная интегральная проводимость слоя равна сумме составляющих проводимости, учитывающих объемные и поверхностные столкновения носителей заряда. Суммируя (20), (23), (25) и (29) и проведя серию математических упрощений, получим следующее выражение для интегральной проводимости:

$$
\begin{gathered}
\sigma_{a}\left(x_{0}, y_{0}, \beta_{0}, q_{1}, q_{2}\right)=a \sigma_{0} \Sigma\left(x_{0}, y_{0}, \beta_{0}, q_{1}, q_{2}\right) \\
\begin{aligned}
& \Sigma\left(x_{0}, y_{0}, \beta_{0}, q_{1}, q_{2}\right)= \frac{x_{0}}{z_{0}}\left(1-\frac{3}{32 z_{0}} B_{1}-\frac{3}{16 \pi z_{0}} B_{2}\right. \\
&\left.-\frac{3}{2 \pi z_{0}} B_{3}\right) \\
& B_{1}=\Theta\left(\beta_{0}-2\right) \int_{-1}^{1} g_{1}\left(z_{0} \varphi_{1} / \beta_{0}\right) d \eta+\Theta\left(2-\beta_{0}\right) \\
& \quad \int_{-1}^{\beta_{0}-1} g_{1}\left(z_{0} \varphi_{1} / \beta_{0}\right) d \eta
\end{aligned}
\end{gathered}
$$

$$
B_{2}=\Theta\left(2-\beta_{2}\right) \int_{\beta_{0}-1}^{1} F\left(\frac{\beta_{0}}{\eta+1}\right) g_{1}\left(z_{0} \varphi_{1} / \beta_{0}\right) d \eta
$$




$$
\begin{gathered}
B_{3}=\Theta\left(2-\beta_{0}\right) \int_{\beta_{0} / 2}^{1} \int_{\beta_{0} / \rho-1}^{1} \rho^{2} \sqrt{1-\rho^{2}} g_{2}\left(z_{0} \varphi_{2} / \beta_{0}\right) d \eta d \rho \\
F(\xi)=\arcsin \xi-\xi\left(1-2 \xi^{2}\right) \sqrt{1-\xi^{2}}, \\
g_{1}(p)=\frac{2-q_{1}-q_{2}-\left(q_{1}+q_{2}-2 q_{1} q_{2}\right) \exp (-p)}{\left[1-q_{1} \exp (-p)\right]\left[1-q_{2} \exp (-p)\right]} \\
\quad \times[1-\exp (-p)], \\
g_{2}(p)=\frac{2-q_{1}-q_{2}+\left(q_{1}+q_{2}-2 q_{1} q_{2}\right) \exp (-p)}{1-q_{1} q_{2} \exp (-2 p)} \\
\times[1-\exp (-p)], \\
\varphi_{1}-\varphi_{d}^{\prime}=2 \pi-\arccos \eta, \\
\varphi_{2}=\varphi_{u d}^{\prime}=\pi-\arccos \eta-\arccos \left(\beta_{0} / \rho-\eta\right), \\
\eta=\xi \beta_{0} / \rho+\cos \alpha .
\end{gathered}
$$

Здесь $\Sigma-$ безразмерная проводимость слоя, которая зависит от следующих безразмерных параметров: толщина слоя $x_{0}$, частота электрического поля $y_{0}$, индукция магнитного поля $\beta_{0}$ и коэффициентов зеркальности верхней и нижней поверхности $q_{1}$ и $q_{2}$.

Найдем составляющие проводимости, учитывающие только объемное и только поверхностное рассеяние носителей заряда. Выражение, определяющее проводимость с учетом объемного рассеяния (29), упрощается и принимает следующий вид:

$$
\begin{gathered}
\sigma_{a v}\left(x_{0}, y_{0}, \beta_{0}\right)=a \sigma_{0} \Sigma_{v}\left(x_{0}, y_{0}, \beta_{0}\right), \\
\Sigma_{v}\left(x_{0}, y_{0}, \beta_{0}\right)= \\
\frac{x_{0}}{z_{0}}\left\{1-\Theta\left(2-\beta_{0}\right) F_{v}\left(\frac{\beta_{0}}{2}\right)\right. \\
\left.-\Theta\left(\beta_{0}-2\right) \frac{3 \pi}{8 \beta_{0}}\right\},
\end{gathered}
$$

$F_{v}(\xi)=\left(1-\xi^{2}\right)^{\frac{3}{2}}+\frac{3}{8}\left(\frac{1}{\xi} \arcsin \xi-\left(1-2 \xi^{2}\right) \sqrt{1-\xi^{2}}\right)$.

Здесь $\Sigma_{v}-$ безразмерная проводимость с учетом объемного рассеяния носителей заряда.

Проводимость, учитывающая только поверхностные столкновения носителей заряда, определяется как разность между полной проводимостью и проводимостью с учетом объемных столкновений:

$$
\begin{gathered}
\sigma_{a s}\left(x_{0}, y_{0}, \beta_{0}, q_{1}, q_{2}\right)=a \sigma_{0} \Sigma_{s}\left(x_{0}, y_{0}, \beta_{0}, q_{1}, q_{2}\right), \\
\Sigma_{s}\left(x_{0}, y_{0}, \beta_{0}, q_{1}, q_{2}\right)= \\
\Sigma\left(x_{0}, y_{0}, \beta_{0}, q_{1}, q_{2}\right) \\
-\Sigma_{v}\left(x_{0}, y_{0}, \beta_{0}\right) .
\end{gathered}
$$

Здесь $\Sigma_{s}-$ безразмерная проводимость, обусловленная поверхностными столкновениями носителей заряда.

\section{3. Предельные случаи}

Рассмотрим предельный случай отсутствия магнитного поля $\left(\beta_{0}=0\right)$. В этом случае второе и третье слагаемые в выражении (34) будут равны нулю, остается неравным нулю только первое и четвертое слагаемые. Отметим, что в выражении (40) в экспоненте $\exp \left(-z_{0} \varphi_{2} / \beta_{0}\right)$ имеет место неопределенность. Раскрывая эту неопределенность по правилу Лопиталя, получим

$$
\lim _{\beta_{0} \rightarrow 0}\left(\frac{z_{0} \varphi_{2}}{\beta_{0}}\right)=\frac{z_{0}}{\rho \sin \alpha} .
$$

С учетом (49) получаем следующее выражение для безразмерной проводимости:

$$
\begin{aligned}
\Sigma\left(x_{0}, y_{0}, q_{1}, q_{2}\right)= & \frac{x_{0}}{z_{0}}\left\{1-\frac{3}{2 \pi z_{0}} \int_{0}^{1} \int_{0}^{\pi} \rho^{2} \sqrt{1-\rho^{2}}\right. \\
& \left.\times g_{2}\left(\frac{z_{0}}{\rho} \sin \alpha\right) d \alpha d \rho\right\} .
\end{aligned}
$$

Перейдя от цилиндрической к сферической системе координат в пространстве скоростей $\left(v, \theta, \alpha^{\prime}\right.$, где $\theta-$ азимутальный угол, $\alpha^{\prime}-$ полярный угол), получим выражение для безразмерной проводимости в отсутствие магнитного поля

$$
\begin{aligned}
& \Sigma\left(x_{0}, y_{0}, q_{1}, q_{2}\right)=\frac{x_{0}}{z_{0}}\left\{1-\frac{3}{4 \pi z_{0}} \int_{0}^{1} \gamma\left(1-\gamma^{2}\right)\right. \\
& \times \frac{2-q_{1}-q_{2}+\left(q_{1}+q_{2}-2 q_{1} q_{2}\right) \exp \left(-z_{0} / \gamma\right)}{1-q_{1} q_{2} \exp \left(-2 z_{0} / \gamma\right)} \\
& \left.\quad \times\left[1-\exp \left(-z_{0} / \gamma\right)\right] d \gamma\right\} .
\end{aligned}
$$

Здесь введено обозначение $\gamma=\cos \theta$.

Выражение (51) совпадает с результатом работы [23]. Рассмотрим случай сильного магнитного поля $\left(\beta_{0} \gg 1\right)$. В этом случае третье и четвертое слагаемые в выражении (34) и второе слагаемое в выражении (35) равны нулю.

Безразмерная проводимость принимает следующий вид:

$$
\begin{aligned}
& \Sigma\left(x_{0}, y_{0}, \beta_{0}, q_{1}, q_{2}\right)=\frac{x_{0}}{z_{0}}\left\{1-\frac{3}{32 z_{0}}\right. \\
& \times \int_{-1}^{1}\left[1-\exp \left(-z_{0} \varphi_{1} / \beta_{0}\right)\right] \\
& \left.\times \frac{2-q_{1}-q_{2}-\left(q_{1}+q_{2}-2 q_{1} q_{2}\right) \exp \left(-z_{0} \varphi_{1} / \beta_{0}\right)}{\left[1-q_{1} \exp \left(-z_{0} \varphi_{1} / \beta_{0}\right)\right]\left[1-q_{2} \exp \left(-z_{0} \varphi_{1} / \beta_{0}\right)\right]} d \eta\right\} .
\end{aligned}
$$

При условии $\beta_{0} \gg 1$ выражение $z_{0} \varphi_{1} / \beta_{0}$, стоящее в экспоненте, является малым числом. Разложив в 
первом множителе подынтегрального выражения (52) экспоненты в ряд до первого порядка малости, а во втором - до второго порядка и проведя упрощения, получим следующее выражение:

$$
\begin{aligned}
\Sigma\left(x_{0}, y_{0}, \beta_{0}, q_{1}, q_{2}\right) \approx & \frac{x_{0}}{z_{0}}\left[1-\frac{3 \pi}{8 \beta_{0}}+\frac{3 z_{0}\left(\pi^{2}-4\right)}{8 \beta_{0}^{2}}\right. \\
& \left.\times\left(2 B+1-\frac{B}{A}\right)\right], \\
A= & \left(1-q_{1}\right)\left(1-q_{2}\right), \\
B= & q_{1}+q_{2}-2 q_{1} q_{2} .
\end{aligned}
$$

Отметим, что первое и второе слагаемые в выражении (53) определяют составляющую безразмерной проводимости, учитывающую только объемное рассеяние электронов. В самом деле, в предельном случае сильных магнитных полей второе слагаемое в выражении (45), содержащем тета-функцию $\Theta\left(2-\beta_{0}\right)$, равно нулю. Получаем следующее выражение:

$$
\Sigma_{v}\left(x_{0}, y_{0}, \beta_{0}\right)=\frac{x_{0}}{z_{0}}\left(1-\frac{3 \pi}{8 \beta_{0}}\right) .
$$

Третье слагаемое в выражении (53) описывает безразмерную проводимость с учетом поверхностных столкновений носителей заряда. Заметим, что при больших магнитных полях $\left(\beta_{0} \gg 1\right)$ проводимость практически полностью определяется составляющей $\Sigma_{v}$, поскольку третье слагаемое в выражении (53) имеет больший порядок малости по сравнению со вторым.

В случае, когда толщина слоя много больше длины свободного пробега носителей заряда $a \gg \lambda$, экспоненты в выражениях (39) и (40) быстро затухают. Также слагаемые с множителем $1 / z_{0}$ в выражении (34) стремятся к нулю. Получаем выражение для классической высокочастотной электропроводности толстого слоя [33]:

$$
\sigma_{a}=a \sigma_{0} x_{0} / z_{0}=a \sigma_{0} /(1-i \omega \tau) .
$$

Рассмотрим случай зеркального отражения электронов от поверхностей слоя $\left(q_{1}=q_{2}=1\right)$. В этом случае числитель в выражениях (39) и (40) обращается в нуль. Отсюда следует, что остается не равным нулю только первое слагаемое в выражении (34). Как и в предыдущем предельном случае, получаем выражение для классической высокочастотной электропроводности толстого слоя (57). Данный результат очевиден, поскольку зеркальная поверхность не влияет на функцию распределения носителей заряда.

\section{4. Анализ результатов}

На рис. 3 изображены зависимости безразмерной интегральной проводимости тонкого металлического слоя от безразмерной индукции магнитного поля $\beta_{0}$ в случае постоянного электрического поля $\left(y_{0}=0\right)$.

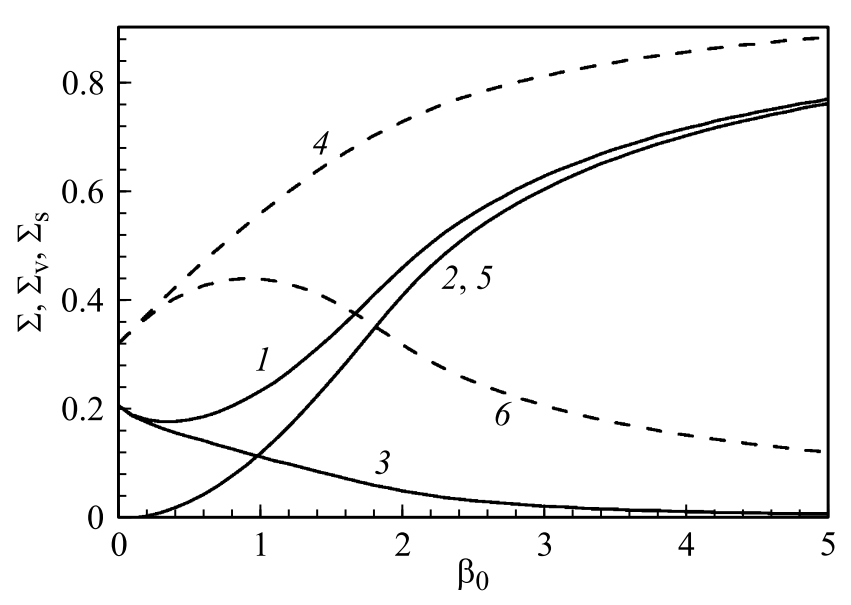

Рис. 3. Зависимости безразмерной интегральной проводимости $\Sigma$ тонкого металлического слоя от безразмерной индукции магнитного поля $\beta_{0}$ в случае постоянного электрического поля $\left(y_{0}=0\right)$ при значениях безразмерной толщины слоя $x_{0}=0.1$ и коэффициентов зеркальности поверхностей $q_{1}=q_{2}=0$ (сплошные кривые $1-3$ ) и $q_{1}=0, q_{2}=1$ (штриховые кривые 4-6): 1,4 - полная безразмерная интегральная проводимость (33); 2, 5 и $3,6-$ составляющие безразмерной проводимости с учетом только объемных $\left(\Sigma_{v}\right)(44)$ и поверхностных $\left(\Sigma_{s}\right)(47)$ столкновений носителей заряда соответственно.

Сплошные кривые построены при одинаковых коэффициентах зеркальности поверхностей $\left(q_{1}=q_{2}=0\right)$, а штриховые кривые - при различных $\left(q_{1}=0, q_{2}=1\right)$. На рис. 3 также построены зависимости составляющих полной безразмерной проводимости слоя, учитывающих объемное $\left(\Sigma_{v}\right)$ и поверхностное $\left(\Sigma_{s}\right)$ рассеяние носителей заряда. На рисунках видно, что с увеличением индукции магнитного поля проводимость, рассчитанная с учетом только объемных столкновений носителей заряда, возрастает, а проводимость, рассчитанная с учетом только поверхностных столкновений, - уменьшается. Это связано с увеличением относительного числа не участвующих в поверхностных столкновениях носителей заряда. Также видно, что в области слабых магнитных полей $\left(\beta_{0}<1\right)$ и при различных коэффициентах зеркальности (штриховая кривая 6) поверхностей наблюдается незначительное увеличение $\Sigma_{s}$. Это можно объяснить следующим образом. В отсутствие магнитного поля все носители заряда движутся по прямолинейной траектории и рассеиваются на обеих поверхностях слоя. При относительно слабых магнитных полях $\left(\beta_{0}<1\right)$ в связи с искривлением траектории носителей заряда появляется группа носителей заряда, рассеивающихся только на одной (верхней или нижней) поверхности слоя. Если одна из поверхностей зеркальная (кривая 6), то в этом случае появляются носители заряда, рассеивающиеся только на зеркальной поверхности слоя. С увеличением магнитного поля относительное число таких носителей заряда по отношению к общему числу участвующих в поверхностных столкновениях носителей 


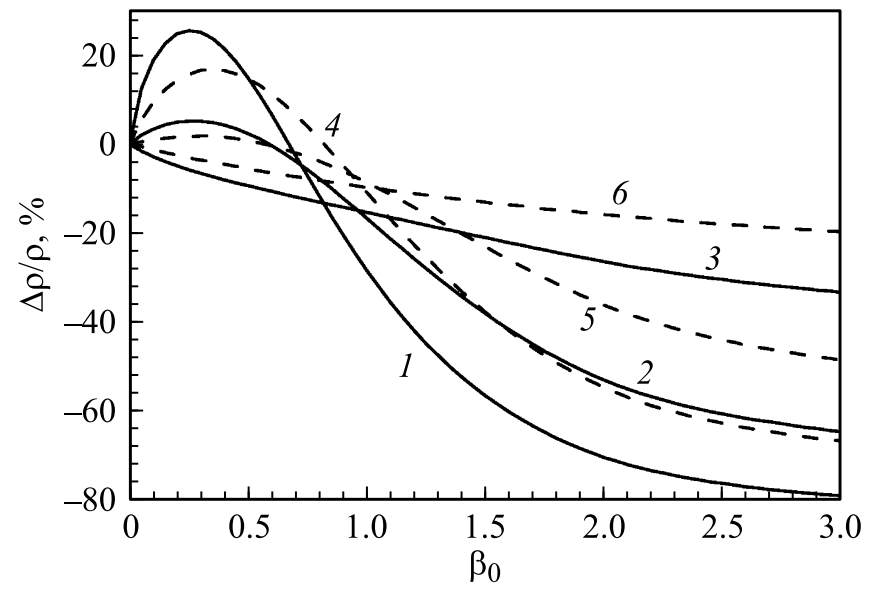

Рис. 4. Зависимости относительного магнетосопротивления $\Delta \rho / \rho$ тонкого металлического слоя (57) от безразмерной индукции магнитного поля $\beta_{0}$ в случае стационарного электрического поля $\left(y_{0}=0\right)$ при значениях безразмерной толщины слоя $x_{0}$, равной 0.05 (сплошные кривые $1-3$ ) и 0.1 (штриховые кривые 4-6): $1,4-q_{1}=q_{2}=0 ; 2,5-q_{1}=q_{2}=0.5 ; 3,6-$ $q_{1}=q_{2}=0.9$.

заряда возрастает. Этим объясняется увеличение составляющей $\Sigma_{s}$ с ростом индукции магнитного поля при $\beta_{0}<1$ в случае одной диффузной и одной зеркальной поверхности. При больших магнитных полях $\left(\beta_{0}>1\right)$ количество рассеивающихся в объеме слоя носителей заряда, определяющих составляющую проводимости $\Sigma_{v}$, становится существенным. При значениях $\beta_{0}>1$ наблюдается уменьшение $\Sigma_{s}$ как в случае одинаковых, так и в случае различных коэффициентов зеркальности поверхностей.

На рис. 4 построены зависимости относительного магнетосопротивления тонкого металлического слоя $\Delta \rho / \rho$ от индукции магнитного поля $\beta_{0}$ в случае постоянного электрического поля $\left(y_{0}=0\right)$. Относительное магнетосопротивление рассчитывалось по следующей формуле:

$$
\Delta \rho / \rho=\left(\rho_{B}-\rho_{0}\right) / \rho_{0}=\Sigma_{0} / \Sigma_{B}-1
$$

где $\rho_{B}, \rho_{0}-$ соответственно удельные сопротивления при наличии и отсутствие магнитного поля, $\Sigma_{B}$ и $\Sigma_{0}-$ безразмерные проводимости при наличии и отсутствие магнитного поля. Безразмерная толщина слоя $x_{0}$ равна 0.05 (сплошные кривые 1-3) и 0.1 (штриховые кривые 4-6). На рисунке видно, что при малых магнитных полях магнетосопротивление растет, достигает максимума при значениях индукции магнитного поля, равных 0.25 (сплошные кривые) и 0.4 (штриховые кривые), затем убывает. С увеличением коэффициентов зеркальности поверхности максимальное значение магнетосопротивления уменьшается, а при коэффициентах зеркальности, больших 0.5, магнетосопротивление становится отрицательным. С увеличением безразмерной толщины пленки максимальное значение магнетосопро- тивления уменьшается и смещается в сторону больших значений $\beta_{0}$.

На рис. 5 изображены зависимости безразмерной проводимости тонкого металлического слоя от коэффициента зеркальности верхней поверхности $q_{2}$ в случае постоянного электрического поля $\left(y_{0}=0\right)$ при различных значениях безразмерной индукции магнитного поля. Коэффициент зеркальности нижней поверхности одинаковый и равен единице. Наблюдается монотонное уменьшение проводимости с уменьшением $q_{2}$. При значениях $q_{1}=q_{2}=1$, отвечающим случаю зеркальных поверхностей, все кривые сходятся в одну точку. Это означает, что зеркальная поверхность не влияет на функцию распределения носителей заряда, т. е. наблюдается переход к классическому результату для проводимости макроскопического образца.

На рис. 6 построены зависимости модуля и аргумента полной безразмерной проводимости тонкого металлического слоя от безразмерной индукции магнитного поля $\beta_{0}$. Безразмерная частота электрического поля равна единице. Сплошные кривые построены при одинаковых коэффициентах зеркальности, а штриховые кривые - при разных. При совпадении безразмерных частоты электрического и индукции магнитного полей $\left(y_{0}=\beta_{0}=1\right)$ и нулевых значениях коэффициентов зеркальности наблюдается резонансно-подобное явление: модуль проводимости резко возрастает, а аргумент принимает такое же значение, как в отсутствие магнитного поля. Резонанс аргумента проводимости достигается при значении $\beta_{0}=0.5$. С увеличением коэффициентов зеркальности максимум модуля и аргумента проводимости смещается в сторону более слабых магнитных полей. При значениях $q_{1}=0$ и $q_{2}=1$ максимум модуля про-

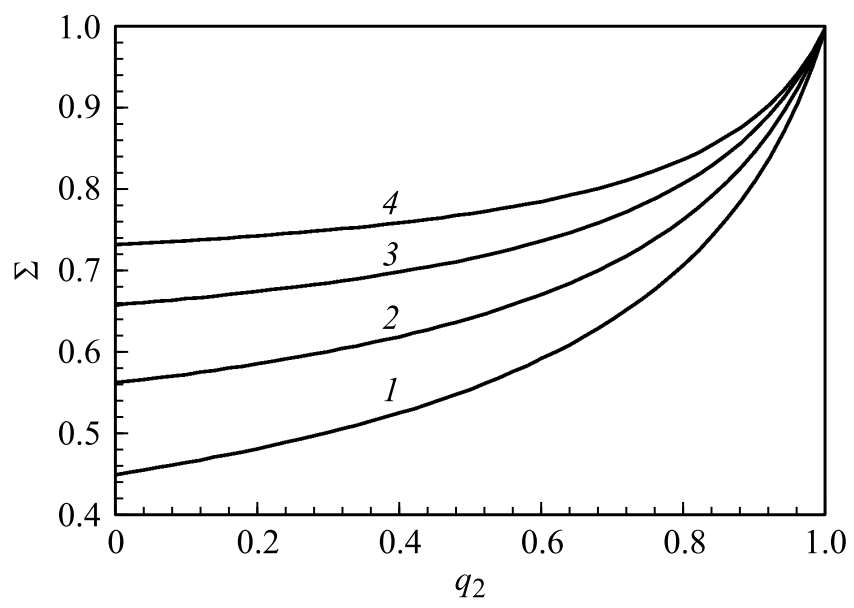

Рис. 5. Зависимости безразмерной интегральной проводимости $\Sigma$ тонкого металлического слоя от коэффициента зеркальности верхней поверхности $q_{2}$ в случае стационарного электрического поля $\left(y_{0}=0\right)$ при значениях безразмерной толщины слоя $x_{0}=0.1$, коэффициента зеркальности нижней поверхности $q_{1}=1$ и различных значениях безразмерной индукции магнитного поля: $1-\beta_{0}=0.5 ; 2-\beta_{0}=1 ; 3-$ $\beta_{0}=1.5 ; 4-\beta_{0}=2$. 

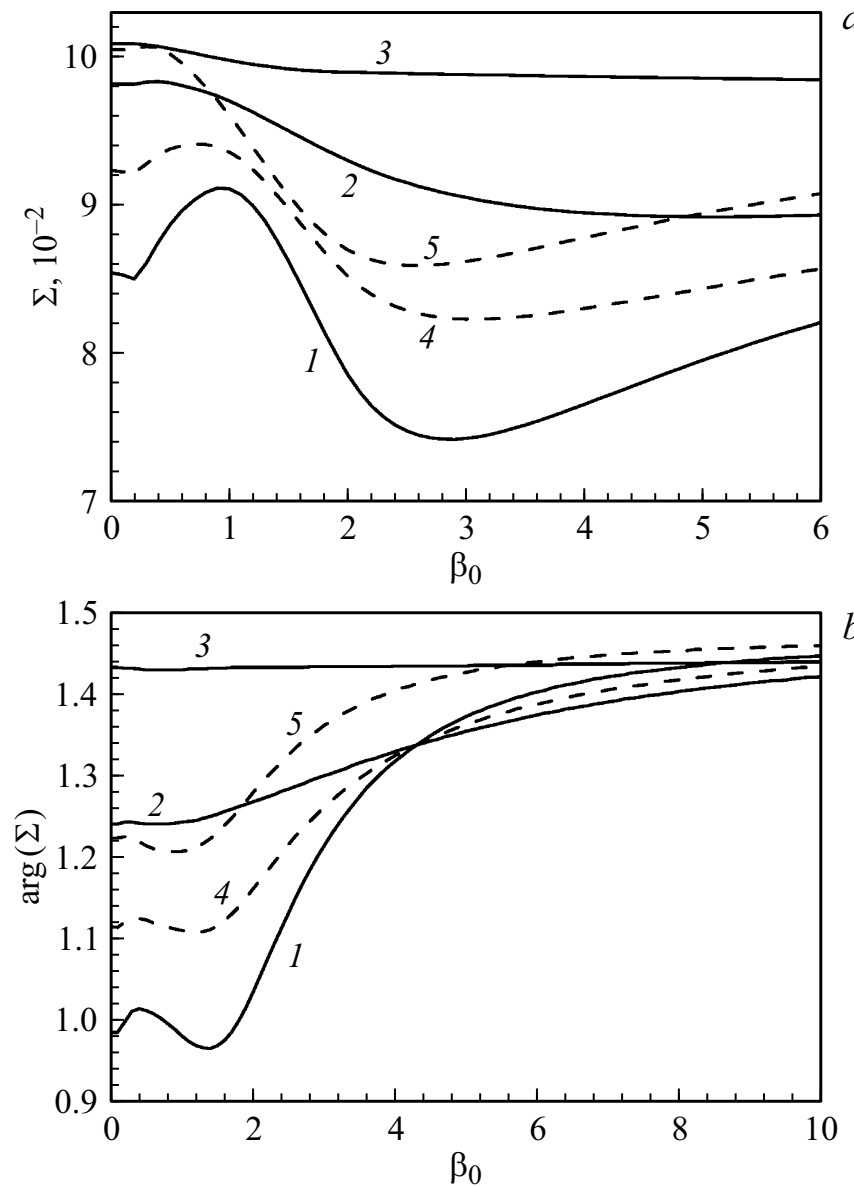

Рис. 6. Зависимости модуля $(a)$ и аргумента $(b)$ безразмерной интегральной проводимости $\Sigma$ тонкого металлического слоя от безразмерной индукции магнитного поля $\beta_{0}$ при значениях безразмерных частоты электрического поля $y_{0}=1$ и толщины слоя $x_{0}=0.1$ : сплошные кривые $1-3: 1-q_{1}=q_{2}=0$; $2-q_{1}=q_{2}=0.5 ; 3-q_{1}=q_{2}=0.9$; штриховые кривые 4,5 : $4-q_{1}=0, q_{2}=0.5 ; 5-q_{1}=0, q_{2}=1$.

водимости находится при значении $\beta_{0}=0.5$ (кривая 5 на рис. $6, a)$, т. е. при значениях $\beta_{0}$, в два раза меньших, чем у кривой 1 , отвечающей случаю $q_{1}=q_{2}=0$.

На рис. 7 построены зависимости безразмерного удельного сопротивления $\rho / \rho_{0}\left(\rho_{0}=m / n e^{2} \tau\right)$ тонкого металлического слоя от безразмерной толщины $x_{0}$. На рис. 7 видно, что с уменьшением толщины слоя сопротивление возрастает. Кривые 1-4 на рис. 7 соответствуют различным коэффициентам зеркальности. Из графиков видно, чем меньше коэффициент зеркальности поверхности, тем выше располагается кривая. Таким образом, рост влияния поверхностных эффектов приводит к увеличению сопротивления слоя. При больших значениях $x_{0}$, независимо от характера отражения носителей заряда от границы слоя, наблюдается стремление к макроскопической асимптотике (57).

На рис. 8 изображены теоретические и экспериментальные зависимости удельного сопротивления трех тонких пленок меди от толщины при температуре
298 К. Точки - экспериментальные данные работы [34], сплошные кривые - теоретический расчет (формулы (33)-(34)). В эксперименте использовалась одна монокристаллическая и две поликристаллические пленки со средними размерами зерен 400 и $200 \mathrm{~nm}$ соответственно. Согласие теоретических расчетов и экспериментальных данных наблюдается при нулевых значениях коэффициентов зеркальности и следующих значениях длин свободного пробега носителей заряда в объеме образца: $38.5 \mathrm{~nm}$ для монокристаллической пленки, $35.2 \mathrm{~nm}$ для поликристаллической пленки с размером

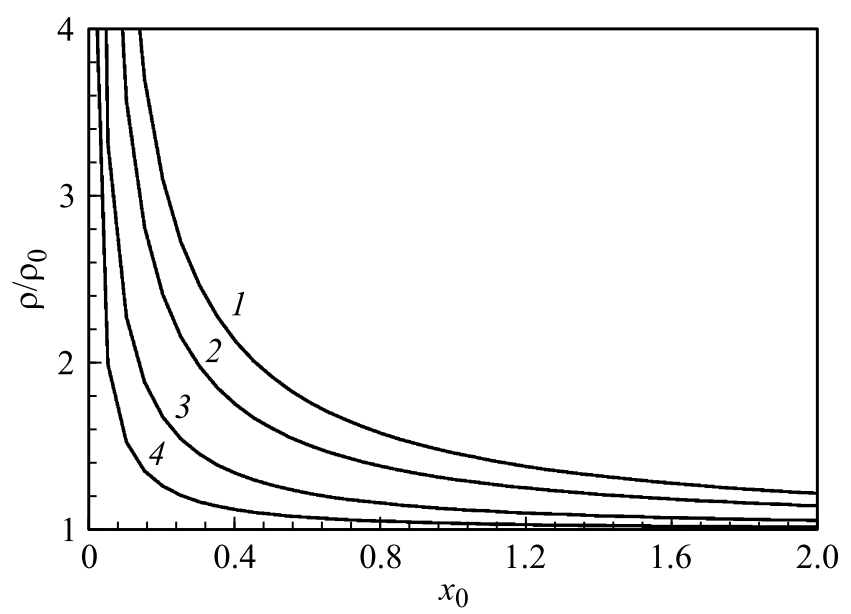

Рис. 7. Зависимости безразмерного сопротивления $\rho / \rho_{0}$ тонкого металлического слоя от безразмерной толщины $x_{0}$ при значениях безразмерных индукции магнитного поля $\beta_{0}$ и частоты электрического поля $y_{0}$, равных нулю: $1-q_{1}=q_{2}=0$; $2-q_{1}=q_{2}=0.3 ; 3-q_{1}=q_{2}=0.7 ; 4-q_{1}=q_{2}=0.9$.

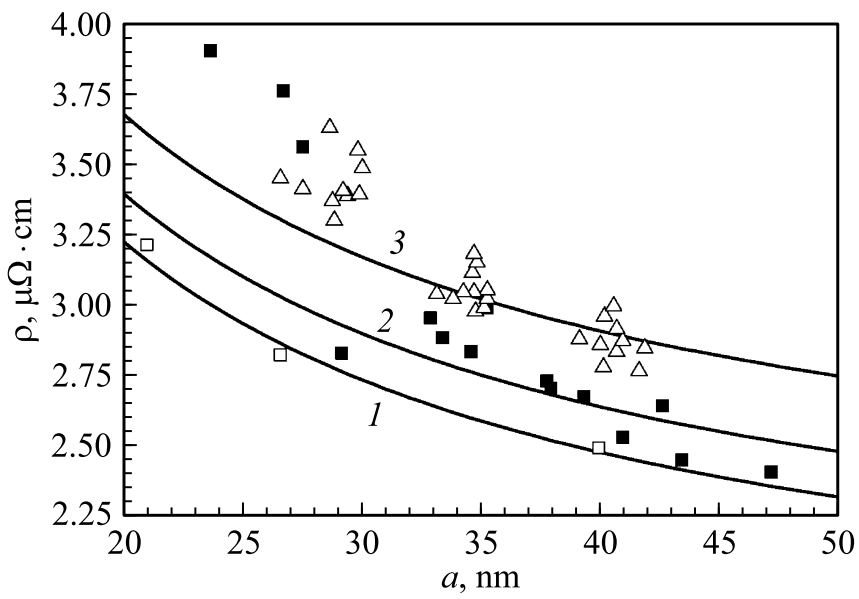

Рис. 8. Зависимости удельного сопротивления трех тонких пленок меди от толщины при температуре 298 К. Точки - экспериментальные данные работы [34] ( $\square$ - сплошная пленка, - - зернистая пленка с размером зерна $400 \mathrm{~nm}, \Delta$ - зернистая пленка с размером зерна $200 \mathrm{~nm}$ ). Сплошные кривые 1 3 - теоретический расчет (33) при значениях $y_{0}=\beta_{0}=0$, нулевых значениях коэффициентов зеркальности поверхностей и следующих значениях длины свободного пробега носителей заряда: $\lambda, \mathrm{nm}: 1-38.5,2-35.2,3-30.6$. 
зерна $400 \mathrm{~nm}$ и $30.6 \mathrm{~nm}$ для поликристаллической пленки с размером зерна $200 \mathrm{~nm}$. Из рис. 8 следует, что в поликристаллических пленках длина свободного пробега меньше, чем в монокристаллической. Возможной причиной уменьшения длины свободного пробега является дополнительное рассеяние носителей заряда на границах зерен.

\section{Заключение}

В работе получены аналитические выражения для интегральной проводимости тонкого металлического слоя, как функции толщины слоя, частоты электрического поля, индукции магнитного поля и коэффициентов зеркальности поверхностей. Анализ зависимостей магнетосопротивления от индукции магнитного поля показывает, что положительное магнетосопротивление наблюдается при значениях коэффициентов зеркальности, меньших 0.5. С увеличением толщины слоя максимальное значение магнетосопротивления уменьшается и смещается в сторону более сильных магнитных полей.

Проанализированы зависимости модуля и аргумента безразмерной проводимости от индукции магнитного поля. Показано, что при диффузном рассеянии носителей заряда и совпадении частоты электрического и индукции магнитного поля наблюдается резонансно-подобное явление для модуля проводимости. Резонанс аргумента проводимости наблюдается при значении безразмерной индукции магнитного поля, в два раза меньшим значения частоты электрического поля.

Проведено сравнение теоретических расчетов с экспериментальными данными для одной монокристаллической и двух поликристаллических пленок меди. Согласие теоретических расчетов с экспериментальными данными наблюдается при нулевых коэффициентах зеркальности поверхностей. Показано, что длина свободного пробега носителей заряда в поликристаллических пленках меньше длины свободного пробега носителей заряда в монокристаллической пленке.

\section{Конфликт интересов}

Авторы заявляют, что у них нет конфликта интересов.

\section{Список литературы}

[1] A. Kowsar, S.F.U. Farhad, S.N. Sakib. IJRER, 8, 2218 (2018).

[2] B. Godefroid, G. Kozyreff. Phys. Rev. Appl., 8 (034024), 1 (2017). https://doi.org/10.1103/PhysRevApplied.8.034024

[3] S. Bhattacharya, I. Baydoun, M. Lin, S. John. Phys. Rev. Appl., 2019. 11 (014005), 1 (2019). https://doi.org/10.1103/PhysRevApplied.11.014005

[4] Л.С. Лунин, М.Л. Лунина, А.С. Пащенко, Д.Л. Алфимова, Д.А. Арустам, А.Е. Казакова Письма в ЖТФ, Т. 45 (6), 7 (2019). https://doi.org/10.21883/PJTF.2019.06.47489.17635 [L.S. Lunin, M.L. Lunina, A.S. Pashchenko, D.L. Alfimova, D.A. Arustamyan, A.E. Kazakova. Tech. Phys. Lett., 45 (6), 250 (2019). https://doi.org/10.1134/S1063785019030313]
[5] А.Б. Никольская, М.Ф. Вильданова, С.С. Козлов, О.И. Шевалеевский. ФТП, 52 (1), 93 (2018). https://doi.org/10.21883/FTP.2018.01.45325.8591

[A.B. Nikolskaia, M.F. Vildanova, S.S. Kozlov, O.I. Shevaleevskiy. Semiconductors, 52 (1), 88 (2018). https://doi.org/10.1134/S1063782618010165]

[6] Ал.А. Никитин, Ан.А. Никитин, А.Б. Устинов, Е. Lahderanta, Б.А. Калиникос. ЖТФ. 86 (6), 115 (2016). [A.A. Nikitin, A.B. Ustinov, E. Lähderanta, B.A. Kalinikos. Tech. Phys. 61 (6), P. 913 (2016).] https://doi.org/10.1134/S106378421606013X

[7] Д.А. Усанов, А.В. Скрипаль, М.К. Мерданов, В.О. Горлицкий. ЖТФ, 86 (2), 65 (2016). [D.A. Usanov, A.V. Skripal', M.K. Merdanov, V.O. Gorlitskii. Tech. Phys., 61 (2), 221 (2016).] https://doi.org/10.1134/S1063784216020250

[8] L. Wang, M. Yin, A. Khan, S. Muhtadi, F. Asif, E.S. Choi, T. Datta. Phys. Rev. Appl., 9 (024006), 1 (2018). https://doi.org/10.1103/physrevapplied.9.024006

[9] M.A. Abeed, J.L. Drobitch, S. Bandyopadhyay. Phys. Rev. Appl., 11 (054069), 1 (2019).

[10] А.И. Ансельм. Введение в теорию полупроводников. (Наука, М., 1978, 616 с.)

[11] И.М. Лифшиц, М.Я. Азбель, М.И. Каганов. Электронная теория металлов. (Наука, М., 1971, 415 с.)

[12] K. Fuchs. Proc. Camb. Phil. Soc., 34, 100 (1938). https://doi.org/10.1017/S0305004100019952

[13] E.H. Sondheimer. Adv. Phys., 50, 499 (2001). https://doi.org/10.1080/00018730110102187

[14] Y.S. Way, Y.H. Kao. Phys. Rev. B., 5, 2039 (1972). https://doi.org/10.1103/PhysRevB.5.2039

[15] R.G. Chambers. Proc. Roy. Soc. Lond. A., 202, 378 (1950). https://doi.org/10.1098/rspa.1950.0107

[16] J. Peterseim, G. Thummes, H.H. Mende. Phys. Stat. Sol. (a), 59, K25 (1980). https://doi.org/10.1002/pssa.2210590160

[17] V. Kuckhermann, G. Thummes, H.H. Mende. Phys. Stat. Sol. (a), 73, 439 (1982). https://doi.org/10.1002/pssa.2210730218

[18] A.A. Yushkanov, O.V. Savenko, I.A. Kuznetsova. Physicascripta, 95 (045805), (2020). https://dx.doi.org/10.1088/1402-4896/ab635a

[19] L. Moraga, C. Arenas, R. Henriquez, S. Bravo, B. Solis. Physica B: Condens. Matter., 499, 17 (2016). https://doi.org/10.1016/j.physb.2016.07.001

[20] R. Henriquez, S. Oyarzun, M. Flores, M.A. Suarez, L. Moraga, G. Kremer, C.A. Gonzalez-Fuentes, M. Robles, R.C. Munoz. J. Appl. Phys., 108 (123704), 1 (2010). https://doi.org/10.1063/1.3525704

[21] R.C. Munoz, M.A. Suárez, S. Oyarzún. Phys. Rev. B, 81 (165408), 1 (2010). https://doi.org/10.1103/PhysRevB.81.165408

[22] S. Oyarzún, R. Henríquez, M.A. Suárez, L. Moraga, G. Kremer, R.C. Munoz. Appl. Surf. Sci. 289, 167 (2014). https://doi.org/10.1016/j.apsusc.2013.10.128

[23] А.И. Уткин, Э.В. Завитаев, А.А. Юшкановю Поверхность. Рентгеновские, синхротронные и нейтронные исследования. 9, 85 (2016). https://doi.org/10.7868/S0207352816090158

[A.I. Utkin, E.V. Zavitaev, A.A. Yushkanov. J. Surf. Invest.: X-Ray, Synchrotron and Neutron Techniques, 10, 962 (2016). https://doi.org/10.1134/S1027451016050153]

[24] А.И. Уткин, А.А. Юшканов. Микроэлектроника, 45 (5), 386 (2016). https://doi.org/10.7868/S0544126916050100 [A.I. Utkin, A.A. Yushkanov. Russ. Microelectronics, 45, 357 (2016). https://doi.org/10.1134/S1063739716050103] 
[25] А.И. Уткин, А.А. Юшканов. ЖТФ, 86 (10), 15 (2016). [A.I. Utkin, A.A. Yushkanov. Tech. Phys., 61 (10), 1457 (2016). https://doi.org/10.1134/S1063784216100273]

[26] И.А. Кузнецова, О.В. Савенко, А.А. Юшканов. ЖТФ, 87 (12), 1769 (2017). https://doi.org/10.21883/JTF.2017.12.45196.1831

[I.A. Kuznetsova, O.V. Savenko, A.A. Yushkanov. Tech. Phys., 62 (12), 1766 (2017). https://doi.org/10.1134/S1063784217120143]

[27] И.А. Кузнецова, Д.Н. Романов, А.А. Юшканов. Микроэлектроника, 47 (3), 226 (2018). https://doi.org/10.7868/S0544126918030079 [I.A. Kuznetsova, D.N. Romanov, A.A. Yushkanov. Russ. Microelectronics, 47 (3), 201 (2018). https://doi.org/10.1134/S1063739718030071]

[28] А.И. Уткин, А.А. Юшканов. Опт. спектр., $\mathbf{1 2 4}(2)$, 250 (2018). https://doi.org/10.21883/OS.2018.02.45532.19017 [A.I. Utkin, A.A. Yushkanov. Opt. Spectr., 124 (2), 247 (2018). https://doi.org/10.1134/S0030400X18020194]

[29] А.И. Уткин, А.А. Юшканов. Поверхность. Рентгеновские, синхротронные и нейтронные исследования. 3, 62 (2019). https://doi.org/10.1134/S0207352819030181 [A.I. Utkin, A.A. Yushkanov. J. Surf. Invest.: X-Ray, Synchrotron and Neutron Techniques, 13, 221 (2019). https://doi.org/10.1134/S1027451019020186]

[30] Э.В. Завитаев, К.Е. Харитонов, А.А. Юшканов. ЖТФ, 89 (5), 643 (2019).

https://doi.org/10.21883/JTF.2019.05.47462.275-18

[E.V. Zavitaev, K.E. Kharitonov, A.A. Yushkanov. Tech. Phys., $64(5), 593$ (2019).

https://doi.org/10.1134/S1063784219050268]

[31] I.A. Kuznetsova, D.N. Romanov, A.A. Yushkanov. Phys. Scr., 94 (115805), (2019).

https://doi.org/10.1088/1402-4896/ab20e4

[32] И.А. Кузнецова, О.В. Савенко, А.А. Юшканов. Микроэлектроника, 45 (2), 126 (2016).

https://doi.org/10.7868/S0544126916020071

[I.A. Kuznetsova, O.V. Savenko, A.A. Yushkanov. Russ. Microelectronics, 45 (2) 119 (2016). https://doi.org/10.1134/S1063739716020074]

[33] Л.Д. Ландау, Е.М. Лифшиц. Теоретическая физика. Т. 8. (Физматлит, М, 2005. 656 c.)

[34] J.S. Chawla, F. Gstrein, K.P. O’Brien, J.S. Clarke, D. Gall. Phys. Rev. B, 84 (235423), 1 (2011). https://doi.org/10.1103/PhysRevB.84.235423 